\title{
Erratum
}

Ann Lab Med 2021;41:128

(A) https://doi.org/10.3343/alm.2021.41.1.128

Check tor
updates

ISSN 2234-3806 elSSN 2234-3814

\section{ANNALS OF

\section{Erratum: Human Platelet Antigen Datasets for Malays, Chinese, and Indians in Peninsular Malaysia}

This erratum is being published to correct the name of a co-author in the article titled "Human Platelet Antigen Datasets for Malays, Chinese, and Indians in Peninsular Malaysia" and authored by Che Ghazali Norul Hajar, Zulkifli Zefarina, Nor Suhaila Md. Riffin, Tuan Hulwani Tuan Mohammad, Mohd Nazri Hassan, Ameer Mohamed Dafalla, Gehad ElGhazali, Geoffrey Keith Chambers, and Hisham Atan Edinur (Ann Lab Med 2020;40:493-9, https://doi.org/10.3343/alm.2020.40.6.493).

\section{Before correction:}

Che Ghazali Norul Hajar, M.DSc. ${ }^{1}$, Zulkifli Zefarina, M.D., M.Path. ${ }^{2}$, Nor Suhaila Md. Riffin, M.D., M.Path. ${ }^{3}$, Tuan Hulwani Tuan Mohammad, M.D., M.Path. ${ }^{4}$, Mohd Nazri Hassan, M.D., M.Path. ${ }^{2}$, Ameer Mohamed Dafalla, Ph.D. ${ }^{5}$, Gehad ElGhazali, M.D., M.Path. ${ }^{6}$, Geoffrey Keith Chambers, Ph.D. ${ }^{7}$, and Hisham Atan Edinur, Ph.D. ${ }^{1}$

\section{After correction:}

Che Ghazali Norul Hajar, M.DSc. ${ }^{1}$, Zulkafli Zefarina, M.D., M.Path. ${ }^{2}$, Nor Suhaila Md. Riffin, M.D., M.Path. ${ }^{3}$,

Tuan Hulwani Tuan Mohammad, M.D., M.Path. ${ }^{4}$, Mohd Nazri Hassan, M.D., M.Path. ${ }^{2}$, Ameer Mohamed Dafalla, Ph.D. ${ }^{5}$, Gehad ElGhazali, M.D., M.Path. ${ }^{6}$, Geoffrey Keith Chambers, Ph.D. ${ }^{7}$, and Hisham Atan Edinur, Ph.D. ${ }^{1}$ 